kommt, wo ihr Geldwerth bis jetzt viel grösser ist, als ihrem Nährwerth entsprechen dürfte.

\title{
Zur Verbreitung des Zinkes im Pflanzenreiche.
}

\author{
Von
}

\section{Laband.}

Mittheilung aus dem Laboratorium für angewandte Chemieder Kgl. Universität München.

Was das Vorkommen des Zinkes in Pflanzen und seine schädliche Wirkung für dieselben anlangt, so liegen darüber zahlreiche Untersuchungen vor. M. Fre ita $\left.{ }^{1}\right)$ fand, dass alle Pflanzen dieses Element aufnehmen, wenn es Ihnen geboten ist, ohne in ihrem Wachsthum gestört zu werden. Die Samen enthielten nur sehr geringe Mengen Zink. Demgegenüber kommt Freitag ${ }^{2}$ ) selbst zu dem Ergebnisse, dass Zink schädlich einwirkt und gewisse Veränderungen der Pflanze, wenn nicht ihr Zugrundegehen bedingt. Dies fanden auch $\mathrm{C}$. Krauch und Andere bestätigt.

C. $\mathrm{Krauch}^{3}$ ), der über Pflanzenvergiftungen arbeitete, berichtet über Gerste-Wasserkulturen, bei welchen der Nährlösung $0,1-0,8 \mathrm{~g}$ Zinksulfat $\left(\mathrm{ZnSO}_{4}+7 \mathrm{H}_{2} \mathrm{O}\right)$ auf ein Liter beigegeben war. Diese lieferten das Ergebniss, dass alle Pflanzen erkrankten und zu Grunde gingen. Nur diejenigen, welche $0,1 \mathrm{mg}$ Zink erhalten hatten, fristeten sich bis in den Herbst kümmerlich fort. C. K ra u ch bespricht sodann den schädlichen Einfluss der zinkhaltigen Abwässer von Olsberg.

F. Nobbe, P. Baesler und H. Willat) prüften das Verhalten von Zinknitrat und -karbonat gegen Pflanzen und fanden, dass bei einem Gehalt von 1/1000 Zink diese nach drei Tagen verkïmmerten.

Besonders eingehende Untersuchungen hat Anton Bauman $n^{5}$ ) angestellt und zwar Vegetationsversuche: 1. mit Nährstofflösungen, welche bestimmte Mengen Zinksulfat enthielten, 2. im Boden, der mit Zinklösung begossen wurde und endlich im Boden, welchem unlösliche Zinksalze zugesetzt waren.

Aus den Vegetationsversuchen geht hervor, dass die Wirkung des Zinksulfates weit schädlicher ist, als angenommen wurde. Lösungen, die 0,1 mg Zink im Liter enthalten, sind unschädlich, bei $1 \mathrm{mg}$ gedeihen die Pflanzen noch. Die Schädlichkeitsgrenze liegt zwischen 1-5 mg Zink im Liter. Ferner spielt auch die Widerstandsfähigkeit eine gewisse Rolle, insofern als ältere Pflanzen gleicher

1) Vierteljahresschrift für praktische Pharm. 19, 427.

2) Jahresber. Agric-Chem. 26, 208.

3) Journal für Landwirthschaft 1882, 30, 271.

$\left.{ }^{4}\right)$ Landw. Vers.-Stat. 1883, 30, 381.

5) Landw. Vers.-Stat. 1884, 31, 1. 
Art eher absterben als jüngere; die Wirkung des Zinkes macht sich durch auffallende Veränderung des Blattgrüns bemerkbar.

Die Vegetationsversuche mit Futterpflanzen im Boden, der mit Zinksulfatlösung begossen wurde, ergaben, dass die Pflanzen im kalk- und humusarmen Sandboden zu Grunde gehen, während sie im humösen Kalkboden keinen Schaden leiden. Die Erklärung dieser Erscheinung ist in der ungleichen Absorptionsfähigkeit der verschiedenen Böden für Zinksalze zu suchen. Die Absorptionsfähigkeit der reinen Humusböden ist am stärksten, dann folgen Thon- und Kalkböden und schliesslich arme Sandböden.

Obgleich man vermuthen sollte, dass Zinkkarbonat wie -sulfid, da sie in kohlensäurereichem Wasser ziemlich löslich sind, ebenfalls schädlich werden können, so haben doch die Versuche ergeben, dass beide Salze im Boden ohne Bedeutung für die auf ibm erzeugte Vegetation sind. Der Verfasser stellte durch Versuche fest, dass weder das Sulfid noch das Karbonat im Boden den Pflanzen zu schaden vermögen, weil die Löslichkeit in kohlensäurehaltigem Wasser durch gleichzeitig vorhandenes Calciumkarbonat vermindert, bei Zinksulfid sogar völlig aufgehoben wird.

Wie ist nun mit den vorliegenden Untersuchungen der mitunter nicht unbedeutende Gehalt an Zink in den Pflanzen in Einklang zu bringen?

Obwohl die Schädlichkeitsgrenze, wie es obige Untersuchungen ergeben, bei $1 \mathrm{mg}$ Zink im Liter liegt, so kommen doch in der Natur Pflanzen vor, die bedeutend mehr von diesem Elemente aufzuweisen vermögen, ohne daran zu Grunde zu gehen.

So scheint dieses Element nach den Beobachtungen Risse's ${ }^{1}$ ) in allen Pflanzen vorzukommen, welche um Altenberg bei Aachen auf zinkreichem Boden wachsen. Es enthielten daselbst in Procenten der Trockensubstanz Thlaspi alpestre in der Wurzel 0,167, in dem Stengel 0,385 und in den Blättern 1,50\% Zinkoxyd. Auch wurde Zink von Forchham mer ${ }^{2}$ ) in dem Holze einiger Bäume, von Lechartier und Bellamy ${ }^{3}$ ) in dem Samen einiger Pflanzen in Spuren nachgewiesen. Eine Veilchenart, welche den Namen "Galmeiveilchen" (Viola lutea var. multicaulis) führt, verändert in zinkfreiem Boden ihre Form nicht, und ebenso hat die Kultur in zinkhaltigem Boden auf die Gestalt von Viola tricolor und Thlaspi alpestre keinen Einfluss, so dass nach den Untersuchungen von $H . H o f f m a n n^{4}$ ) das von $R$ isse beobachtete besonders üppige Gedeihen von Silene inflata und Armeria gerade auf dem zinkreichsten Boden nicht einem direkten Einfluss dieses Metalles entspringen.

Ueber ein anderes Beispiel von der grossen Aufnahmefähigkeit des Zinkes durch die Pflanzen berichtet Reichhardt ${ }^{5}$ ).

1) Mitgetheilt in Sachs "Experimentalphysiologie".

2) Annal. Chem. u. Physik 95, 89.

${ }^{3}$ ) Compt. rend. 84, 688.

4) Botan. Zeitung 1875, 628; 1877, 36 .

$\left.{ }^{5}\right)$ Jahresber. Agric.-Chem. 10, 139. 
Aus Versehen wurde 1 Pfund ganz koncentrirte syrupdicke Zinkchloridlösung zum grössten Theil auf einen im Kübel stehenden grossen Oleanderbaum, 7um kleineren auf ein Agapanthus-Exemplar gegossen. Der Boden war sehr reich an Kalk. Die direkt angegriffenen Theile des Agapanthus starben ab, die übrigen erbielten sich. Der Oleander verlor eine Menge Blätter, entwickelte aber gleichzeitig viel junge Triebe, die aber ein viel helleres Grün zeigten.

Die Analyse ergab $z w e i$ bis drei Tage nach dem Vorfall in den abgefallenen Blättern $0,1436 \%$ und in einem grünen Aste mit Blättern $0,664 \%$ Zinkoxyd.

Die später ausgeführten Untersuchungen ergaben ferner in Rinde, Holz, Stengeln und Blättern folgenden Gehalt an Zinkoxyd:

$\begin{array}{lccc} & \text { nach } 8 \text { Tagen } & \text { nach } 6-7 \text { Wochen } & \text { im nächsten Frühjahr } \\ \text { Rinde } & 1,066 \% & 0,271 \% & 0,330 \% \\ \text { Holz } & 0,500 \% & 0,283 \% & 0,385 \% \\ \text { Stengel } & - & - & 0,346 \% \\ \text { Blätter } & - & 0,214, & 0,406 \%\end{array}$

E. Jensch ${ }^{1}$ ) hat endlich ausfïhrlich iiber seine Beobachtungen und Arbeiten iiber die Galmeiflora von Oberschlesien berichtet. Seine interessanten Mittheilungen stellen die Thatsache fest, dass die auf zinkhaltigem Boden gewachsenen Pflanzen (Tussilago Farfara, Polygonum aviculare) hohen Feuchtiggehalt, sowie grosse Aschenmengen aufweisen, ferner der procentige Gehalt der Aschen der erwähnten Pflanzen an Zink (auf Zinkkarbonat berechnet) folgender war:

\begin{tabular}{|c|c|c|c|c|c|c|}
\hline & & assilago & Farfara & Polygo & num avi & culare \\
\hline & Wurzel & Blattstiel & Blattscheibe & Wurzeln & Stengel & Blätter \\
\hline lmeihalde I & 2,51 & 1,75 & 2,90 & 1,77 & 2.25 & 1,24 \\
\hline II & 3,26 & 1,63 & 2,83 & 1,93 & 2,86 & 1,49 \\
\hline
\end{tabular}

Am häufigsten beobachtete E. Jensch auf den Galmeihalden ausser den beiden genannten Pflanzen Taracum officinale, Capsella bursa pastoris und Plantago lanceolata.

Ausserdem mögen hier noch die Ergebnisse einer eigenen Untersuchung von auf zinkhaltigem Boden gewachsenen Pflanzen angeführt werden. Die mir durch die Liebenswürdigkeit des Herrn Apotheker Dr. Aschner, dem hier mein Dank dafür ausgesprochen sei, übersandten Pflanzen wuchsen auf dem stark zinkhaltigen Boden von $\mathrm{Sch}$ arley in Oberschlesien in der Nähe der Galmeigruben.

Die Pflanzen, die kein verändertes Aussehen aufwiesen, wurden zunächst gründlich von oberflächlich anhaftendem Sand u. dergl. gereinigt, bei $100^{\circ}$ getrocknet und von dieser Trockensubstanz zwei Portionen von je $100 \mathrm{~g}$ abgewogen. Auch hier erwies sich das von $\mathrm{Halenke} \mathrm{angegebene} \mathrm{Zerstörungsverfahren} \mathrm{mit}$ koncentrirter Schwefelsäure und Quecksilberoxyd als sehr vortheilhaft; nach un-

1) Zeitschr. angew. Chem. 1894, 14. 
gefähr acht Stunden wurde eine farblose Flüssigkeit erhalten, die von der unrerändert gebliebenen Cellulose abfiltrirt wurde. Beim Verdünnen mit Wasser schied sich ein feiner Krystallbrei ab, der sich nach näherer Untersuchung als Calciumsulfat erwies. In die nun schwachsauere Flüssigkeit wurde Schwefelwasserstoff eingeleitet, das ausgefällte Schwefelquecksilber abfiltrirt und der Schwefelwasserstoff auf dem Wasserbade verjagt. Das Zink lässt sich in Fällen, wie in dem vorliegenden, wo einerseits Zink und Eisen, andererseits Calcium und Phosphoräure nebeneinander auftreten, mit den sonst üblichen Reagentien nicht abscheiden. Die Phosphorsäure wurde deshalb nach dem bekannten Verfahren mit Zinn und Salpetersäure herausgeschaffit und dann das Zink aus der essigsauren Lösung mit Schwefelwasserstoff abgeschieden. Der weisslichgraue Niederschlag, der durch etwas Schwefeleisen verunreinigt war, wurde mit Essigsäure ausgewaschen, in Salzsäure gelöst, noch einmal als Zinksulfid ausgefällt und als Zinkoxyd zur Wägung gebracht. In $100 \mathrm{~g}$ der getrockneten. Pflanzen war 0,252 g Zinkoxyd (ZnO) entsprechend 0,2021 g Zink enthalten.

In einer zweiten Probe wurde das Zink elektrolytisch bestimmt. Gefunden wurden $0,2018 \%$ Zink.

Anmerkung. Noch mehr Litteratur über vorstehende Frage, als hier angegeben ist, und eine eingehendere Erklärung des verschiedenen Verhaltens des Zinkes in den Bodenarten findet sich in des Unterzeichneten Werke: "Die Verunreinigung der Gewässer etc.". Berlin 1899. 2. Aufl. II. Bd. 427-439.

J. König.

\section{Ein einfacher Apparat zur gleichzeitigen Bestimmung des Fettes und des Wassers in der Butter.

\author{
Von
} \\ H. Poda, Adjunkt.}

Mittheilung aus der statlichen Untersuchungsanstalt für Lebensmittel iu Graz. (Vorstand: Professor Dr. Prausnitz.)

Durch die vielfachen Verfälschungen der verbreitetsten Nahrungs- und Genussmittel ist das Bedürfniss nach einfachen, schnell arbeitenden Verfahren für eine möglichst genaue Untersuchung immer mehr zu Tage getreten.

Diesem Bedürfniss verdankt auch die im Folgenden zu beschreibende Methode zur schnellen Feststellung des Wasser- und Fettgehaltes der Butter ihr Entstehen. Sie unterscheidet sich von anderen rasch auszuführenden Methoden besonders dadurch, dass die zur Untersuchung verwandte Butter nicht abgewogen zu werden braucht.

Ausführung der Methode.

1. Ein Becherglas von etwa $20 \mathrm{~cm}$ Höhe und $15 \mathrm{~cm}$ Durchmesser oder 